\title{
INFLUÊNCIA DA ADUBAÇÃO COM ESTERCO BOVINO E INOCULAÇÃO DE FUNGOS MICORRÍZICOS ARBUSCULARES NO CRESCIMENTO DE MUDAS DE CARICA PAPAYA L. (VAR. FORMOSA)
}

\author{
Cláudia Elizabete de Lima Lins ${ }^{2}$ \\ Regina Lúcia Félix de Aguiar ${ }^{2}$ \\ Uided Maaze T. Cavalcante ${ }^{2}$ \\ Leonor Costa Maia ${ }^{2}$
}

\author{
Recebido em 28/01/1999. Aceito em 11/08/1999
}

\begin{abstract}
RESUMO - (Influência da adubação com esterco bovino e inoculação de fungos micorrízicos arbusculares no crescimento de mudas de Carica papaya L. (var. Formosa). Devido ao rápido crescimento do mamoeiro, poucos são os solos que, em condições naturais, podem satisfazer a sua demanda por nutrientes, sendo necessária a aplicação de fertilizantes minerais que favoreçam as plantas no período do transplantio para o campo. Os fungos micorrízicos arbusculares (FMA) são conhecidos por tornarem os seus hospedeiros mais resistentes aos estresses bióticos e abióticos, sendo por isso empregados na produção de mudas de diversas árvores frutíferas. Nesse trabalho objetivou-se avaliar a influência da inoculação de FMA e da aplicação de matéria orgânica no crescimento de mudas de mamoeiro (Carica papaya L. var. Formosa), em casa-de-vegetação. Mudas foram cultivadas em solo arenoso (caracterizado pelo baixo teor de P: 4 ppm), inoculado com FMA e adubado ou não com 50g de esterco bovino. O delineamento experimental foi inteiramente casualizado com seis tratamentos e três repetições: - inoculação com FMA nativos, com Gigaspora albida Schenck \& Smith, ou com Scutellospora heterogama (Nicol. \& Gerd.) Walker \& Sanders, com ou sem matéria orgânica. O experimento foi avaliado a cada 10 dias, sendo aferidos os parâmetros: altura, diâmetro do caule e número de folhas. Diferenças significativas entre os tratamentos adubados e não adubados foram observados a partir do $30^{\circ}$ dia. Após 40 dias, as plantas inoculadas com os FMA nativos apresentaram melhor desenvolvimento do que as inoculadas isoladamente com G. albida ou $S$. heterogama, em todos os parâmetros avaliados, tanto em solo adubado como em solo não adubado.
\end{abstract}

Palavras-chave - micorrizas, produção de mudas, mamoeiro

\begin{abstract}
Influence of fertilization with bovine manure and inoculation of arbuscular mycorrhizal fungi in the growth of Carica papaya L. 'Formosa' seedlings). Due to the fast growth of papaya, few are the soils that can satisfy its demand for nutrients under natural conditions, the application of mineral fertilizers that favor the plants in the period of the transplant to the field being necessary. The arbuscular mycorrhizal fungi (AMF) are known for making their hosts more resistant to biotic and abiotic stress, this being the reason why they have been employed for the seedling production of several types of fruit trees. The objectives of this work were to evaluate the influence of the inoculation of AMF and the application of organic matter in the growth of papaya (Carica papaya L. 'Formosa'), in a greenhouse. Seedlings were cultivated in sandy soil (characterized by a low P: 4ppm), inoculated with AMF and fertilized or not with $50 \mathrm{~g}$ of bovine manure. The experimental design was entirely randomized with six treatments and three replicates: - inoculation with either native AMF, Gigaspora albida Schenck \& Smith or Scutellospora heterogama (Nicol. \& Gerd.) Walker \& Sanders with/without organic matter. The experiment was evaluated every 10 days, considering the parameters: height, diameter of stem and number of leaves. Significant differences between fertilized and non fertilized treatments were observed on the 30 th day. After 40 days, plants inoculated with native AMF presented better development than those inoculated separately with $G$. albida or $S$. heterogama, in both fertilized and non fertilized soils.
\end{abstract}

Key words - mycorrhizae, seedling production, papaya

Trabalho apresentado no $49^{\circ}$ Congresso Nacional de Botânica, Salvador, BA

2 Departamento de Micologia, CCB, Universidade Federal de Pernambuco, Av, Prof. Moraes Rego s/nº, CEP 50.670-420, Recife, PE, Brasil e-mail: claudia_lins@hotmail.com 


\section{Introdução}

Os fungos micorrízicos arbusculares (FMA) são de grande importância para o crescimento e nutrição de plantas frutíferas (Weber \& Amorim 1994) e, de acordo com Silva \& Siqueira (1991), as espécies que passam por fase de formação de mudas em viveiros, apresentam vantagens quando inoculadas com FMA. Além de crescerem mais rapidamente, devido aos teores mais elevados de alguns nutrientes, tornam-se mais resistentes aos estresses bióticos e abióticos.

O mamoeiro (Carica papaya L. var. Formosa) é planta frutífera de rápido crescimento que produz, dentro de período relativamente curto, grande safra de frutos. De acordo com estimativas da Food and Agriculture Organization of the United Nations FAO (1994), o Brasil apresentou produção de 1.800 .000 t/ano com participação de aproximadamente $29,03 \%$ na produção mundial e, segundo o IBGE (1995), o Estado de Pernambuco participou com $0,37 \%$ da produção do Nordeste, enquanto a Bahia se destacou como principal produtor do Brasil. Pernambuco apresentou produtividade equivalente a $50 \%$ da obtida nos outros Estados nordestinos. Entretanto, sob condições naturais, poucos são os solos que podem satisfazer a demanda de nutrientes desta fruteira sem a aplicação de fertilizantes (Medina et al. 1980).

Recomenda-se para a cultura a adição de matéria orgânica no solo, sendo que o esterco de curral curtido, o composto orgânico ou os adubos verdes devem ser a base de qualquer tratamento de fertilidade (Jimenez 1957). Essas recomendações têm base empírica, pois foram feitas poucas pesquisas sobre a adubação dessa cultura (Oliveira et al.1994). A micorrização tem sido de grande importância em mudas de mamoeiro por torná-las mais fortes e resistentes ao serem transferidas para o campo (Saggin Jr. \& Siqueira 1996).

Ramirez et al. (1975) verificaram que a inoculação micorrízica favoreceu o crescimento de mamoeiros quando foi adicionada solução de Hoagland ao solo. Silva \& Siqueira (1991) observaram que a produção de matéria seca de mamoeiros foi aumentada pela associação com a maioria dos FMA testados, com acréscimo ou não de superfosfato simples ao substrato. No presente trabalho avaliaram-se os efeitos de FMA nativos e introduzidos e da aplicação de matéria orgânica no crescimento de mudas de mamoeiro var. Formosa em casa-de-vegetação.

\section{Material e métodos}

O solo utilizado foi do tipo franco arenoso, coletado em Aldeia (Camaragibe, Pernambuco) na profundidade entre 15 e $20 \mathrm{~cm}$ e apresentou as seguintes características: $\mathrm{pH}=4,5 ; \mathrm{P}=4,3 \mathrm{ppm}$; $\mathrm{N}=0,1 \mathrm{ppm} ; \mathrm{C}=11,8 \mathrm{~g} / \mathrm{dm}^{3}$ e matéria orgânica $=20,4 \mathrm{~g} / \mathrm{dm}^{3}$. Para utilização no experimento, o solo foi fumigado com brometo de metila.

FMA nativos deste solo e isolados de $G$. albida Schenck \& Smith (International Culture Collection of Arbuscular Mycorrhizal Fungi INVAM-927) e $S$. heterogama (Nicol. \& Gerd.) Walker \& Sanders (EMBRAPA - Centro Nacional de Pesquisa em Agrobiologia e UFPE-12) foram multiplicados em associação com Passiflora edulis Sims F.sp. flavicarpa Deg. (maracujá Amarelo), durante três meses, para serem utilizados como inóculo. A densidade de esporos do solo foi avaliada após peneiramento úmido (Gerdemann \& Nicolson 1963) e centrifugação em sacarose (Jenkins 1964), sendo encontrados 58 esporos/g de solo de $G$. albida e de $S$. heterogama e 1,5 esporos/g de solo para FMA nativos, representados por espécies de Glomus Tul. \& Tul., Acaulospora Gerd. \& Trappe, Gigaspora Gerd. \& Trappe e Scutellospora Walker \& Sanders.

O solo inóculo foi colocado em vasos com capacidade para $500 \mathrm{ml}$, os quais receberam plântulas de mamão, pré-germinadas em solo esterilizado. Metade dos vasos recebeu $450 \mathrm{~g}$ do solo-inóculo e $50 \mathrm{~g}$ de matéria orgânica (esterco bovino), com o restante recebendo $500 \mathrm{~g}$ apenas do substrato inoculado. O delineamento experimental foi inteiramente casualizado, com seis tratamentos e três repetições: F1A0, F1A1, F2A0, F2A1, F3A0 e F3A1, sendo: (F1) solo com FMA nativos, (F2) solo com G. albida, (F3) solo com S. heterogama, (A0) sem adubação orgânica e (A1) com adubação orgânica.

$\mathrm{O}$ experimento teve a duração de 50 dias. Diariamente, utilizando-se termohigrômetro (TFA, Alemanha), foram registrados os valores de 
temperatura e umidade na casa-de-vegetação, que variaram respectivamente de $22^{\circ}$ a $39^{\circ} \mathrm{C}$ e de 35 a $88 \%$. A cada 10 dias foram aferidos: altura das plântulas, diâmetro do caule (a $3 \mathrm{~cm}$ acima do solo) e número de folhas; ao final, foram analisadas a produção da massa seca da parte aérea e a densidade dos esporos por $50 \mathrm{~cm}^{3}$ de solo.

Os dados foram analisados estatisticamente por análise de variância do Programa Statistica (StatSoft 1995).

\section{Resultados e discussão}

As mudas de mamoeiro mantidas em substrato adicionado de matéria orgânica apresentaram maior desenvolvimento, diferenciando-se significativamente das não adubadas, em todos os parâmetros analisados. A presença de FMA aumentou de modo significativo apenas o número de folhas e a massa seca, havendo também interação significativa nestes mesmos parâmetros entre a inoculação e a presença de matéria orgânica (Tab. 1).

Em relação à altura e ao diâmetro do caule das plantas (Fig. 1a e 1b), não houve diferença significativa entre os tratamentos com FMA, enquanto entre substrato adubado e não adubado foi altamente significativo a partir dos 20 dias. Ramirez et al. (1975) observaram que, aos 40 dias, a altura de plântulas de mamoeiro em solo fertilizado com solução de Hoagland, e inoculadas com Scutellospora calospora ou Glomus a )

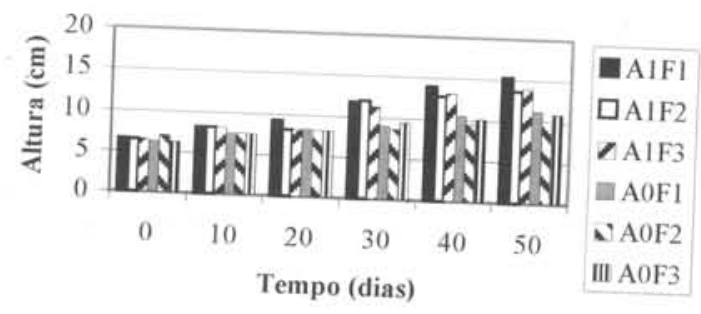

b )

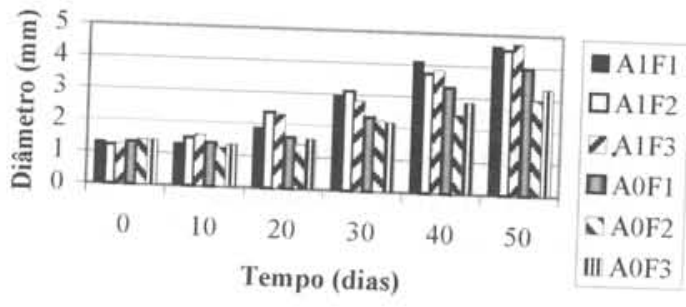

c )

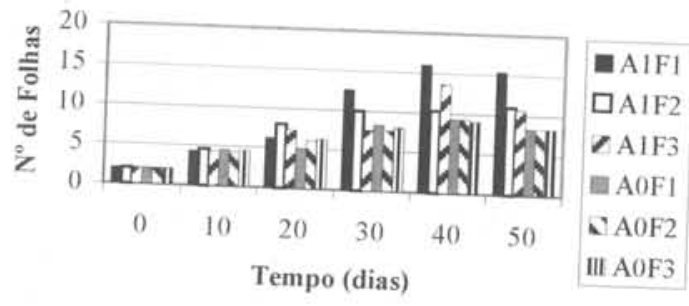

Figura 1. Altura (a), diâmetro do caule a $3 \mathrm{~cm}$ do solo (b) e número de folhas (c) de plantas de mamoeiro 'Formosa' cultivadas em solo adubado (A1) ou não com matéria orgânica (A0), sob diferentes tratamentos de inoculação. FMA nativos (F1), G. albida (F2) e S. heterogama (F3).

Tabela 1. Valores médios de altura, diâmetro do caule, número de folhas e massa da parte aérea seca das plantas de mamoeiro $\mathrm{A}=$ altura das plântulas; $\mathrm{D}=$ diâmetro do caule; $\mathrm{NF}$ = núlo ou não com matéria orgânica, sob diferentes tratamentos de inoculação. (*) Significante para $\mathrm{P}<0,05$, (**) Significante para $\mathrm{P}<0,01$; (NS) Não significante.

\begin{tabular}{|c|c|c|c|c|c|}
\hline \multirow{2}{*}{\multicolumn{6}{|c|}{$\begin{array}{c}\text { Parâmetros avaliados } \\
\text { A }(\mathrm{cm})\end{array}$}} \\
\hline & & $\mathrm{A}(\mathrm{cm})$ & $\mathrm{D}(\mathrm{mm})$ & NF & $\operatorname{MS}(\mathrm{g})$ \\
\hline Al & FMA nativos & 15,3 & 4,66 & 15,3 & 0,39 \\
\hline & G. albida & 13,6 & 4,53 & 11,0 & 0,29 \\
\hline & S. heterogama & 14,0 & 4,73 & 10,6 & 0,30 \\
\hline AO & FMA nativos & 11,3 & 3,96 & 8,33 & 0,29 \\
\hline & G. albida & 10,2 & 2,96 & 8,33 & 0,15 \\
\hline & S. heterogama & 11,0 & 3,33 & 8,33 & 0,17 \\
\hline & Significância & & & & \\
\hline & $\mathrm{S}$ & ** & ** & $* *$ & ** \\
\hline & FMA & NS & NS & * & $* *$ \\
\hline & SX FMA & NS & NS & * & ** \\
\hline
\end{tabular}


macrocarpum foi significativamente mais alta do que as inoculadas com $S$. heterogama, que não apresentou diferença quando comparada ao tratamento controle.

Quanto ao número de folhas, os fungos foram igualmente efetivos no solo não adubado (Fig. 1c), enquanto no solo adubado os FMA nativos sobressaíram em relação a $G$. albida e $S$. heterogama, sem no entanto apresentar diferenças significativas entre si ( F1 $>\mathrm{F} 2=\mathrm{F} 3)$. Aos 40 dias, o tratamento adubado e inoculado com $S$. heterogama atingiu o ponto máximo em número de folhas, e a partir daí começou a declinar. Talvez o tamanho dos vasos utilizados tenha contribuído para esse resultado, impedindo a continuidade do crescimento das raízes devido ao pouco espaço disponível.

A massa seca das plântulas inoculadas com FMA nativos foi estatisticamente diferente da produzida pelas associadas a $G$. albida e $S$. heterogama, tanto em solo adubado como em não adubado, mostrando a eficiência dos FMA nativos frente aos introduzidos (Fig. 2).

Como o mamoeiro requer aplicação de fertilizantes (Medina et al. 1980), é comum encontrar trabalhos que associem a inoculação de FMA à aplicação de adubação correta. Nos estudos de Silva \& Siqueira (1991), a micorrização foi altamente favorável para o crescimento de mamoeiros, com os autores constatando que, mesmo na ausência de adubação fosfática, a inoculação com Glomus clarum, G. macrocarpum, S. heterogama e mistura de FMA, aumentou a produção de matéria seca. Silva \& Siqueira (1991) atribuíram o fato de terem obtido valores da massa seca maiores que os encontrados por Ramirez et al. (1975), a diferenças varietais ou à influência das condições de crescimento, como por exemplo a fertilidade do substrato.

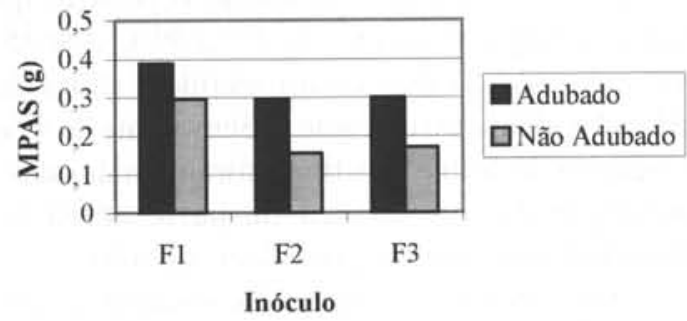

Figura 2. Massa da parte aérea seca (MPAS) após 50 dias de cultivo de plantas de mamoeiro 'Formosa' em solo adubado ou não com matéria orgânica, sob diferentes tratamentos de inoculação. FMA nativos (F1), G. albida (F2) e S. heterogama (F3).

Weber \& Amorim (1994) evidenciaram o benefício dos FMA sobre o crescimento e acúmulo de nutrientes na parte aérea de mamoeiros; com a introdução de Glomus etunicatum e aplicação de 20 ppm de $\mathrm{P}_{2} \mathrm{O}_{5}$, as plantas atingiram valores máximos de altura e produção de matéria seca.

Souza et al. (1991) registraram que mudas de cafeeiro adubadas com matéria orgânica apresentaram maior crescimento, não havendo porém interação significativa entre a inoculação com Gigaspora margarita e matéria orgânica no substrato. Baby \& Manibhushanrao (1996) afirmam que o uso de matéria orgânica pode aumentar a eficiência dos FMA nativos e estes reduzirem a incidência de doenças em arroz.

Durante os 50 dias de experimento, o tratamento adubado e inoculado com FMA nativos apresentou o melhor resultado. Embora a quantidade de esporos de FMA nativos inoculados tenha sido menor que a usada para $G$. albida e $S$. heterogama (Tab.2), os primeiros foram mais eficientes no desenvolvimento de mudas de

Tabela 2. Densidade de esporos de FMA na rizosfera de plântulas de mamoeiro, ao início e ao término do experimento.

\begin{tabular}{l|ccc}
\multicolumn{2}{c}{ FMA } & \multicolumn{3}{c}{ Número de esporos/g solo rizosférico } \\
\hline & Inicial & Não adubado & Adubado \\
FMA nativos & & 48,0 & 26,0 \\
G. albida & 1,50 & 55,0 & 83,0 \\
S. heterogama & 58,0 & 36,0 & 30,0 \\
\hline
\end{tabular}


mamoeiro, independentemente do substrato utilizado. Os experimentos devem prosseguir em condições de campo, visando o aprimoramento de tecnologia utilizando-se FMA nativos para melhor desenvolvimento de mudas de mamoeiros.

\section{Agradecimentos}

À colega Adriana Mayumi Yano de Melo, pelas sugestões e incentivo; à FACEPE, pela concessão de bolsa de Iniciação Científica à primeira Autora; ao CAPES, pela concessão de bolsas de Mestrado e Doutorado, respectivamente, às segunda e terceira Autoras; ao $\mathrm{CNPq}$, pela concessão de bolsa de Produtividade em Pesquisa à quarta Autora.

\section{Referências bibliográficas}

Baby, U. I. \& Manibhushanrao, K. 1996. Influence of organic amendments on arbuscular mycorrhizal fungi in relation to rice sheath blight disease. Mycorrhiza 6(3): 201-206.

Food and Agriculture Organization of the United Nations FAO. 1994. Production Yearbook, v. 48, Rome.

Gerdemann, J. W. \& Nicholson, T. H. 1963. Spores of mycorrhizae Endogone species extracted from soil by wet sieving and decanting. Transactions of the British Mycological Society 46: 235-244.

IBGE. 1995. Anuário Estatístico do Brasil. Rio de Janeiro.

Jenkins, W. R. 1964. A rapid centrifugal-flotation technique for separating nematodes from soil. Plant Disease Report 48(9): 692.

Jimenez, H. 1957. Injertos entre especies de Carica. Agriculture Tropical 7(1): 33-37.
Medina, J. C.; Garcia, J. L. M. ; Salomón, E. A. G.; Vieira, L. F.; Renesto, O. V.; Figueiredo, N. M. S. \& Canto, W. L. 1980. Mamão, da cultura ao processamento e comercialização - Série Frutas Tropicais, vol. 7. ITAL, Campinas.

Oliveira, A. M. G.; Farias, A R. N.; Santos Filho, H. P.; Oliveira, J. R. P.; Dantas, J. L. L.; Santos, L. B. dos; Oliveira, M. de A.; Souza Júnior, M. T.; Silva, M. J.; Almeida, O. A. de; Nickel, O.; Medina, V. M. \& Cordeiro, Z. J. M. 1994. Mamão para exportação: aspectos técnicos da produção. Ministério da Agricultura, do Abastecimento e da Reforma Agrária. Série Publicações Técnicas Frupex. 9. SDR Embrapa.

Ramirez, B. N., Mitchell, D. J. \& Schenck, N. C. 1975. Establishment and growth efects of three vesicular arbuscular mycorrhizal fungi on papaya. Mycologia 67(5): 1039-1041.

Saggin-Júnior, O. J. \& Siqueira, J. O. 1996. Micorrizas arbusculares em cafeeiro. Pp. 203-254. In: J. O. Siqueira (Ed.), Avanços em fundamentos e aplicação de micorrizas. UFLA - DCS, DCF, Lavras

Silva, L. F. C. \& Siqueira, J. O. 1991. Crescimento e teor de nutrientes de mudas de abacateiro, mangueira e mamoeiro sob influência de diferentes espécies de fungos micorrízicos vesículo arbusculares. Revista Brasileira de Ciência do Solo 15: 283-288.

Souza, C. A S.; Siqueira, J. O.; Oliveira, E. \& Carvalho, J. G. 1991. Crescimento e nutrição de mudas de cafeeiro micorrizadas. Efeito da matéria orgânica e superfosfato simples. Pesquisa Agropecuária Brasileira 26(11/12): 1989-2005.

Statsoft. 1995. Statistica for Windows 1995. Vol. I: General Convertions \& Statistics I. Statsoft, Inc., Oklahoma.

Weber, O. B. \& Amorim, S. M. C. 1994. Adubação fosfática e inoculação de fungos micorrízicos vesiculoarbusculares em mamoeiro 'Solo'. Revista Brasileira de Ciência do Solo 18: 187-191. 